\title{
The Trainability Performance of Sudanese Local Dogs Type in Drug Search
}

Selma AM Hosseen and Ammar ME Ibrahim*

The Police Dogs School, African Centre for Veterinary Consulting (ACVC), Sudan

*Corresponding author: Ammar ME Ibrahim, The Police Dogs School, African Centre for Veterinary Consulting (ACVC), Khartoum, Sudan

Submission: 酱 March 23, 2018; Published: 眥 April 09, 2018

\section{Mini Review}

Dogs have been subjected to a huge selection pressures during domestication and that has resulted in a considerable diversity in morphology and behaviour [1,2]. Most law enforcement agencies around the world use wide range of dog breeds for different tasks. Many researches in the field of dogs training indicated that behavioural differences between dog's breeds are less than behavioral differences within the breed $[3,4]$. Factor such as gender [5] and dog human relationship [6,7] and confinement conditions [8] play significant role in determining the behavioural traits and the characteristics of the dog, particularly during the first year of the dog's life [9].

Here we report an interesting study carried out by the school of police dogs in Sudan to assess the capability of special local dog type in drug detection and search. Eleven puppies between 6 to 11 months age were collected from North Kordofan area in Western Sudan where these puppies are usually herd camels and sheep. The puppies were enrolled in training program composed of three training phases. These training phases consisted of adaptation phase (16 weeks) obedience phase (10 weeks) and search and detection phase (10 weeks). The aim of the training program was to enable the puppies adapt and develop social skills in a disciplined and intensive management and to train puppies to search and detect a local drug (Cannabis) which is very common in Sudan.

All puppies were successfully passed the adaptation phase, seven of them succeeded to pass the obedience phase and none of them have managed to pass the search and detection phase. Puppy's performance was scored according to the assessment protocol of the school of police dogs in Sudan with slight modification according to pre-training processes and guidelines of South African police, puppies which fail to pass one phase successfully were not allowed to move to the next phase.

The highest performing individuals ( 3 males and 3 females) were bred together and seven puppies of their offspring were selected to the same training program. The next generation puppies were successfully passed the first and second training phases and four of them successfully passed the final training phase.

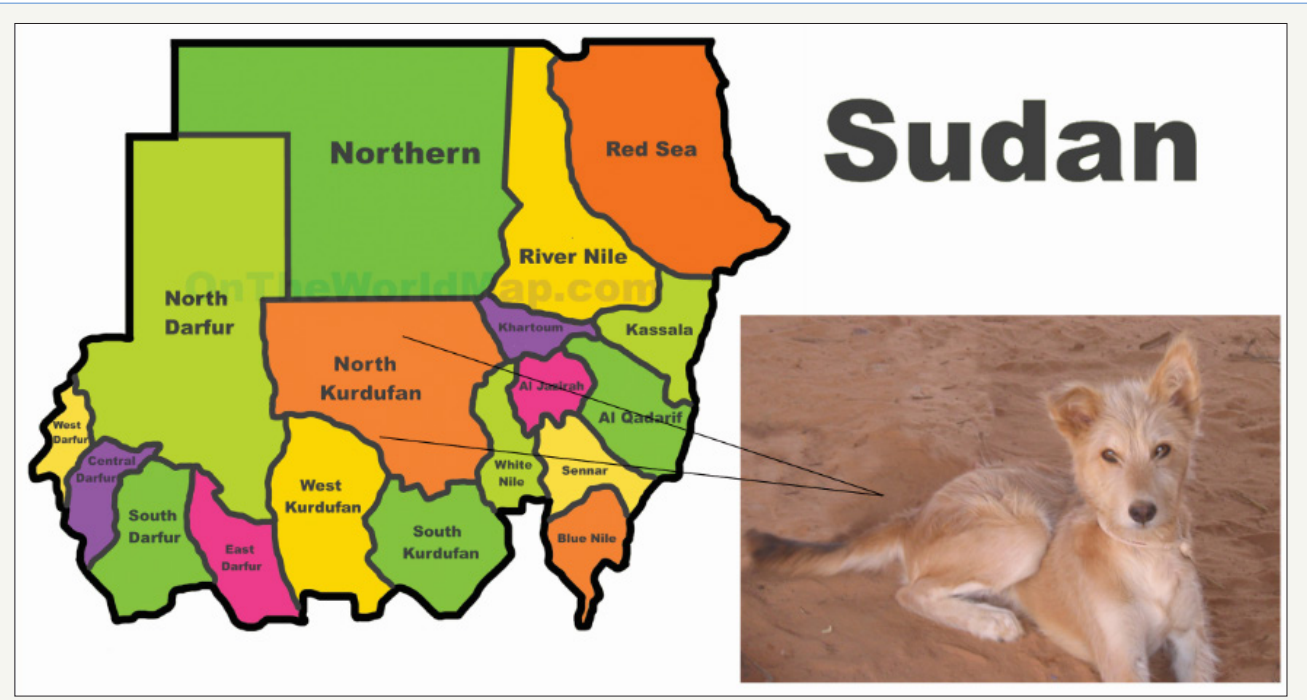

Figure 1: KABABISH DOGS; Local dogs type from North Kurdufan area 
Adaptation phase assessment was divided in to six exercises with total scores (TS) 75, while the mean score (MS) of parent generation was 45 with standard deviation (SD) 7.57, the MS of their off-spring was 61.57 and the SD was 7.06. Seven puppies from the parent's generation managed to pass the next training phase (adaptation phase), The TS was 70 divided into 4 exercises, the MS of parents was 28.57 and the SD was 8.24SD while the performance of their off-spring in the same training phase was 36.14MS with SD 6.56 .

Although none of the parent's generation passed the final training phase (search and detection) which consisted of 5 exercises with TS 60,4 puppies out of $7(\approx 57 \%)$ of their off spring successfully passed the final training phase with MS 40.75 and SD 6.60. The MS for all puppies (7 puppies) was 35.28 and the SD was 8.32 .

The study showed potential capability of this local dog type to search and detect cannabis and possibly other types of drugs. In addition this type of dogs is highly adapted to the climate and land topography where this type of drugs (Cannabis) is cultivated and smuggled. On the other hand the parent generation showed greater differences in their ability to adapt to the confined management system compared to their offspring, this was probably attributed to the age of parents when they were introduced to such breeding and management system which supported Mehrkam \& Wayne [3] and Foyer [9] findings. The study also showed that these dogs are facing difficulties to focus during the exercises and this can be solved by enhancing their social learning skills, particularly by the use of do as I do method described by Fugazza $[10,11]$.

More studies are strongly recommended to support findings and to further the use local dogs in police operations. Police and other law enforcement institutions in the region, need to pay attention to their local dog breeds as the European breeds are not well adapted to the environment where drugs are cultivated and smuggled [12].

\section{Acknowledgment}

The authors would like to thank professor Muntaser Eltayeb Ibrahim and professor Mohamed-Khair Abdallah Ahmed for thier help and guidance in putting this work on a scientifics shape, also our thank extended to Mr. Abdelmonem Idris for providing a high level of professional advice and instruction in the field of dogs training.

\section{References}

1. Cluton BJ (1995) Origins of the dog: domestication and early history. In: Serpell J (Ed.), The domestic dog, its evolution, behaviour and interaction with people. Cambridge: Cambridge University Press, India, pp. 7-20.

2. Miklosi A (2007) Dog behaviour, evolution, and cognition, Oxford: Oxford University Press, USA, pp. 95-130.

3. Mehrkam, Lindsay R (2014) Behavioral differences among breeds of domestic dogs (Canis lupus familiaris): Current status of the science. Applied Animal Behaviour Science 155: 12-57.

4. Svartberg K, Forkman (2002) Personality traits in the domestic dog (Canis familiaris). Applied Animal Behaviour Science 79: 133-155.

5. Hart BL, Hart LA (1985) Selecting pet dogs in the bases of cluster analysis of breed behaviour profile and gender. Journal of the American Veterinary Medical Association 186(11): 1881-1885.

6. Topal JA, Miklson, Csanyi V (1997) Dog human relationship affects problem solving behaviour in the dog. Anthrozoos 10: 214- 224.

7. Pongaracz P, Miklosi A, Kubinyi E, Gurobi K, Topal J, et al. (2001) Social learning in dogs: the effect of a human demonstrator on the performance of dogs in a detour task. Animal Behaviour 62(6): 1109-1117.

8. Borchelt PL, Voith VL (1982) Classification of animal behaviour problems. Veterinary Clinics of North America: Small Animal Practice 12: 571-585.

9. Pernilla F, Bjällerhag N, Wilsson E, Jensen (2014) Behaviour and experiences of dogs during the first year of life predict the outcome in a later temperament test. Applied Animal Behaviour Science 155: 93-100.

10. Fugazza C, Ádám M (2014) Should old dog trainers learn new tricks? The efficiency of the do as I do method and shaping/clicker training method to train dogs. Applied Animal Behaviour Science 153: 53-61.

11. Fugazza C, Ádám M (2015) Social learning in dog training: The effectiveness of the do as i do method compared to shaping/clicker training. Applied Animal Behaviour Science 171: 146-151.

12. Frank H (1980) Evolution of canine information processing under conditions of natural and artificial selection. Z Tierpsychol 53(4): 389399.
Creative Commons Attribution 4.0 International License

For possible submissions Click Here

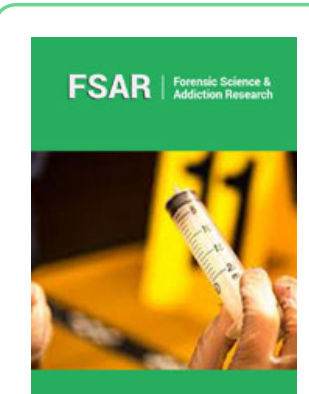

\section{Forensic Science \& Addiction Research}

\section{Benefits of Publishing with us}

- High-level peer review and editorial services

- Freely accessible online immediately upon publication

- Authors retain the copyright to their work

- Licensing it under a Creative Commons license

- Visibility through different online platforms 